\title{
Duodenal mucosal damage is associated with proliferative activity of Brunner's gland hamartoma: a case report
}

\author{
Mayumi Akaki ${ }^{1,2^{*}}$, Shoji Taniguchi ${ }^{3}$, Kinta Hatakeyama ${ }^{4}$, Ryoji Kushima ${ }^{5}$ and Hiroaki Kataoka ${ }^{2}$
}

\begin{abstract}
Background: Brunner's gland hamartoma is a rare tumor, predominantly found in the fifth to sixth decades of life. Generally, it is a single pedunculated polyp, rarely larger than $5 \mathrm{~cm}$. Asymptomatic cases are found incidentally, but cases with a large polyp tend to have gastrointestinal bleeding and/or obstructive symptoms. Polyp size increases in a time-dependent manner, however, the growth mechanism is unknown. We report a Japanese male case in his mid-twenties with an over $6 \mathrm{~cm}$ sized polyp.

Case presentation: A 26-year-old man presented black stools and anemia. Endoscopic examination revealed a large pedunculated polyp at gastroduodenal junction. The polyp, subsequently resected by distal gastrectomy, was lobulated with random surface erosions and sized $6.4 \times 3 \mathrm{~cm}$. Histological examination revealed that the polyp arose from duodenal mucosa and was composed of hyperplastic Brunner's glands in lobules separated by fibromuscular septa, associated with lymphocytic infiltrate and lymphoid follicles. No evidence of malignancy was found. Thus, the lesion was diagnosed as Brunner's gland hamartoma. Further immunohistochemical studies indicated that gastric foveolar metaplasia is associated with surface epithelium covering upper two thirds of the polyp, showing immunohistochemical positivity for mucin 5 AC (MUC5AC). Below the metaplastic surface epithelium, Brunner's glands had high proliferative activity (MIB-1 labeling index: 7.9\%). The similar staining pattern was observed at surface erosive sites (MIB-1 labeling index in Brunner's glands: 9\%). On the other hand, surface epithelium in the lower side of the polyp still preserved intestinal nature, containing CDX2-positive nuclei and MUC2-positive goblet cells. Brunner's glands below the surface epithelium with intestinal characteristics showed low proliferative activity (MIB-1 labeling index: 0.77\%).

Conclusion: Proliferative activity of Brunner's glands was high at the sites with surface erosion and also below the epithelium showing gastric foveolar metaplasia. As gastric foveolar metaplasia occurs along with a mucosal repair process in the duodenum, mucosal damages underlay the hamartomatous proliferation of Brunner's glands and eventually resulted in a formation of large polypoid mass in this case.
\end{abstract}

Keywords: Brunner's gland hamartoma, Brunner's glands, Gastric foveolar metaplasia, MUC5AC, MIB-1, Mucosal damage

\footnotetext{
* Correspondence: mayumi_akaki@med.miyazaki-u.ac.jp

${ }^{1}$ Clinical Laboratory, University of Miyazaki Hospital, 5200 Kihara, Kiyotake, Miyazaki, Miyazaki 889-1692, Japan

2Section of Oncopathology and Regenerative Biology, Department of Pathology, Faculty of Medicine, University of Miyazaki, 5200 Kihara, Kiyotake, Miyazaki, Miyazaki 889-1692, Japan

Full list of author information is available at the end of the article
} 


\section{Background}

Brunner's gland hamartoma is a rare tumor-like lesion, predominantly seen in the fifth to sixth decades of life. This lesion is generally a single pedunculated polyp, with an average size of $2 \mathrm{~cm}$, rarely larger than $5 \mathrm{~cm}$, and locates in the first portion of duodenum [1]. Its pathogenesis is unknown and malignant change is rarely observed [2]. Most cases are asymptomatic and found incidentally. Cases with a large-sized polyp tend to have gastrointestinal bleeding and/or obstructive symptoms. According to studies with long-term follow-up, polyp size gradually increases in a time-dependent manner [3,4].

We experienced a 26 year-old male case with an over $6 \mathrm{~cm}$ sized polyp at gastroduodenal junction, investigated histological and immunohistochemical characteristics of the lesion, and report here.

\section{Case presentation}

A 26-year-old man presented black stools and anemia. He had not taken any medication and had no specific family or past medical history. His height and weight were $172.3 \mathrm{~cm}$ and $93.4 \mathrm{~kg}$ (the body mass index was $31.5 \mathrm{~kg} / \mathrm{m}^{2}$ ). His body temperature was $36.7^{\circ} \mathrm{C}$, blood pressure was $137 / 77 \mathrm{mmHg}$ and radial pulse rate was 80 beats/min and regular. Complete blood count showed the red blood cell count of $326 \times 10^{4} / \mu \mathrm{l}$, hemoglobin concentration of $8.6 \mathrm{~g} / \mathrm{dl}$ (reference range: 13.5 to $17.6 \mathrm{~g} / \mathrm{dl}$ ), and mean corpuscular volume of $87 \mathrm{fl}$. Serum chemistry showed that hemoglobin A1c was 4.2\% (National Glycohemoglobin Standardization Program number). Endoscopic examination of the upper digestive tract revealed a large pedunculated polyp arising from gastroduodenal junction, close to pyloric ring on the side between minor curvature and anterior wall (Figure 1A). The head of the polyp was incarcerated toward duodenal lumen. The lower side of the polyp showed hemorrhage in part. The stalk was too thick to be removed by endoscopic mucosal resection. Instead, distal gastrectomy with lymph node dissection was performed. No metastasis was found in the dissected lymph nodes.

The postoperative period was uneventful that the hemoglobin concentration gradually increased and became $11.6 \mathrm{~g} / \mathrm{dl}$ on day 12 . He was subsequently discharged without any complications and was in good health with 1 year follow-up.

The surgically resected specimen showed a lobulated polyp sized $6.4 \times 3 \mathrm{~cm}$ (Figure 1B) and the cut surface was solid and white (Figure 1C). Histologically, the stalk of the polyp projected from duodenal mucosa, not pyloric mucosa. In the lesion, marked proliferation of Brunner's glands was noted showing lobular structures separated by fibromuscular septa (Figure 2A). Acini and ducts were well preserved (Figure 2C) and cystically dilated ducts were scattered (Figure 2B). Lymphocytic infiltrate
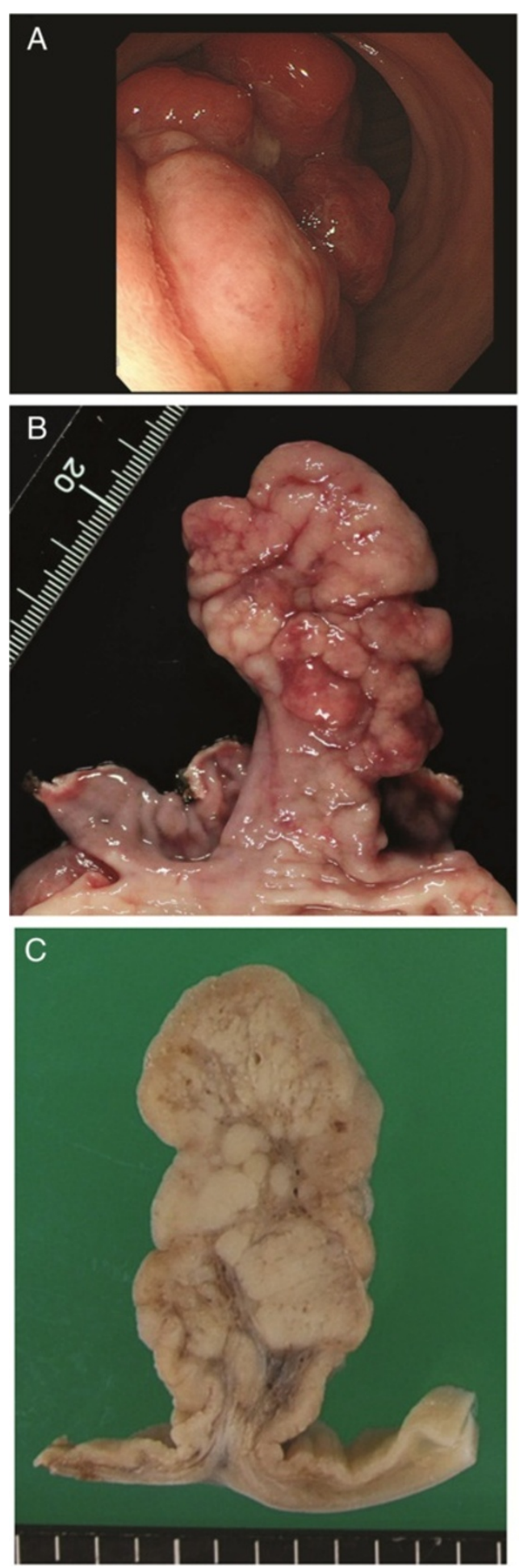

Figure 1 Endoscopic image and gross findings. (A) Endoscopic image showing a large polyp at gastroduodenal junction. The polyp head was incarcerated toward duodenal lumen. Hemorrhage was noted in part of the surface. (B) Surgically resected specimen showing a single lobulated and pedunculated polyp. Hemorrhage and erosion are associated. (C) Cut surface of the polyp is white and solid showing lobular structure and a broad fibrous stalk. 


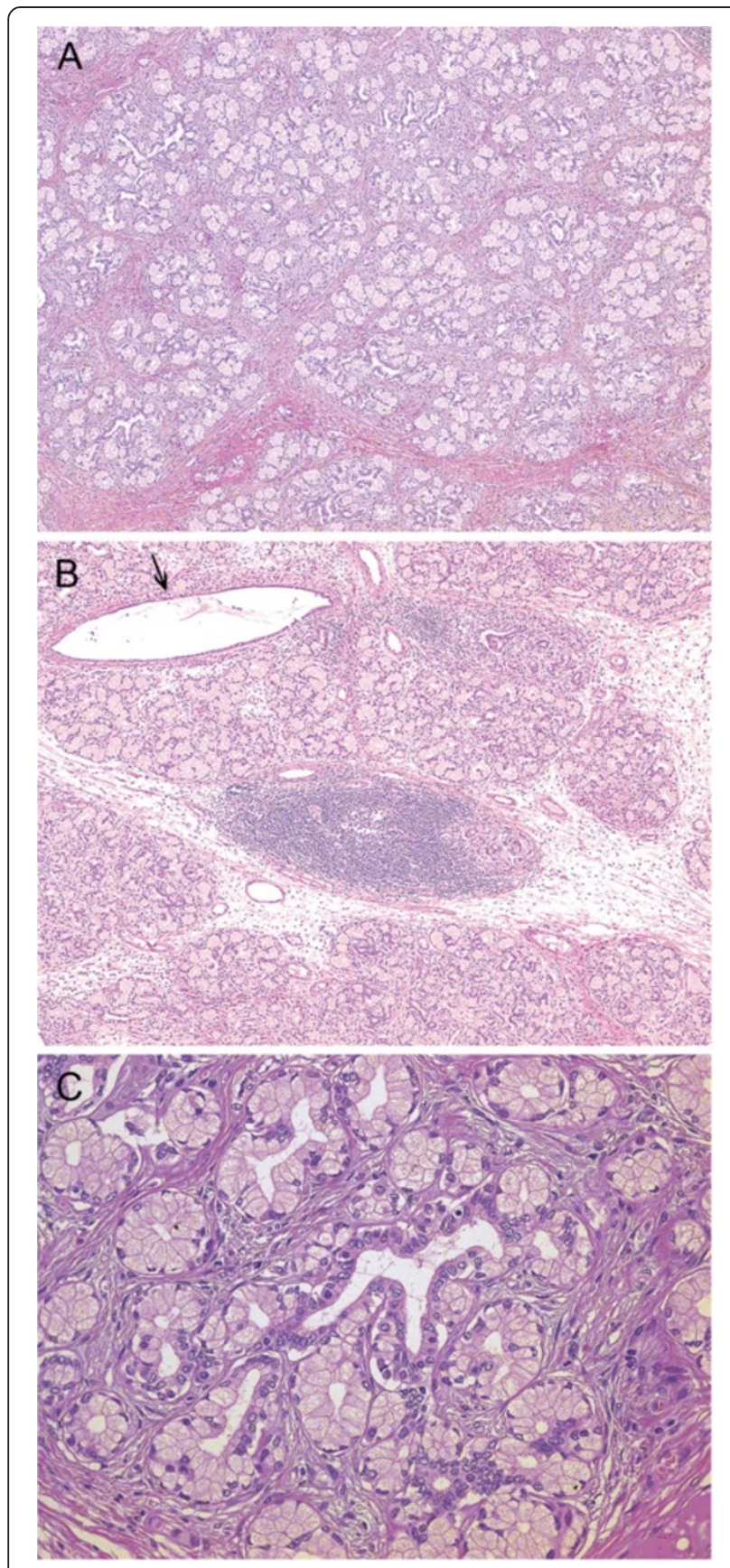

Figure $\mathbf{2}$ Histological findings of the resected specimen.

(A) Hyperplastic Brunner's glands in lobules are separated by fibromuscular septa. HE stain, 40× (B) Lymphocytic infiltrate is observed and a lymphoid follicle is formed between lobules of Brunner's glands at the center. Arrow indicates a cystically dilated duct. HE stain, 40x (C) Acini and ducts are well-preserved without nuclear atypia. HE stain, 200x.

was observed in the entire lesion and lymphoid follicle formation was intermingled in and between the lobules of hyperplastic Brunner's glands (Figure 2B). The upper two thirds of the polyp was covered by a surface epithelium histologically similar to gastric foveolar epithelium (Figure 3A), randomly with surface erosions (Figure 3E). No evidence of malignancy was found within the specimen (Figure 2C). These findings suggested that the lesion was composed of normal tissue elements of duodenal mucosa and submucosa, but formed a disorganized aberrant mass. Thus, we diagnosed this lesion as Brunner's gland hamartoma. Helicobacter pylori infection was not detected in Giemsa stain.

Then, we sought to clarify the possible pathogenesis of this lesion by immunohistochemical analyses for mucin phenotypes, CDX2 expression and MIB-1 labeling. The surface epithelium resembling gastric foveolar epithelium was positive for MUC5AC (Figure 3B), indicating gastric foveolar metaplasia, while the epithelium in deeper portion and Brunner's glands were positive for MUC6 (Figure 3C). The epithelial cells at the transition zone between MUC5AC- and MUC6-positive areas showed concomitant expression of MUC5AC and MUC6 (Figure 3B and $C$ ). These results confirmed that the surface epithelium differentiated towards gastric foveolar epithelium but preserved intestinal epithelial nature in the deeper portion. Interestingly, the MUC5AC-positive epithelium and adjacent MUC6-positive epithelium were diffusely positive for MIB-1 (Figure 3D), and the Brunner's glands beneath them showed high MIB-1 labeling index (7.9\%; Figures 3D and $4 \mathrm{~A})$.

The similar staining pattern was observed in the area with surface erosion (Figure 3E-H), where most of surface epithelium was lost, but the deeper portion of the epithelium was minimally left (Figure $3 \mathrm{E}$ ), showing MUC5AC/MUC6 double positivity (Figure 3F and 3G) with significantly high MIB-1 labeling (Figure $3 \mathrm{H}$ ). Moderately high MIB-1 labeling of Brunner's glands (9\%) was accompanied (Figures $3 \mathrm{H}$ and $4 \mathrm{~B}$ ).

On the other hand, surface epithelium in the lower part of the polyp showed a villous structure containing goblet cells without apparent gastric foveolar metaplasia (Figure 3I). Immunohistochemically, positivity of MUC5AC staining decreased in the lower one third of the polyp, particularly on the distal side. In the same area, CDX2positive nuclei (Figure 3J) and MUC2-positive goblet cells (Figure 3K) were observed at the surface epithelium showing intestinal nature. Below the surface epithelium preserving intestinal nature, Brunner's glands had less proliferative activity with low MIB-1 labeling index $(0.77 \%)$ (Figures $3 \mathrm{~L}$ and $4 \mathrm{C}$ ). These results suggest that gastric foveolar differentiation is possibly related with the observed proliferative activity of Brunner's glands.

\section{Conclusions}

Brunner's gland hamartoma is generally a single pedunculated polyp composed of hyperplastic Brunner's glands with a variable amount of ducts, smooth muscles, fibrous tissue, adipose tissue, and lymphocytes. This lesion is 

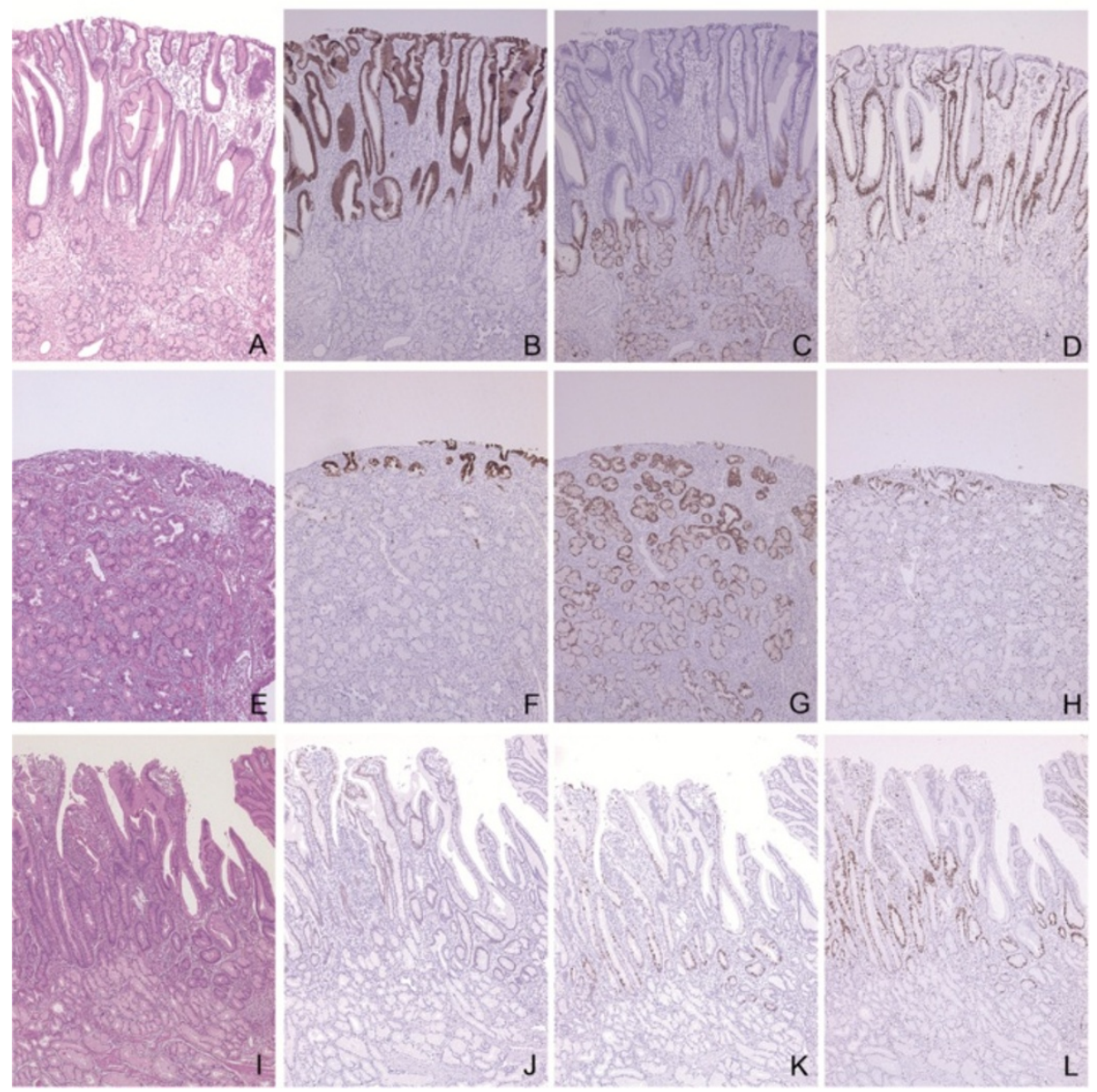

Figure 3 Immunohistochemical findings of the resected specimen. Duodenal mucosa in the upper part of the polyp (A - D), with erosion

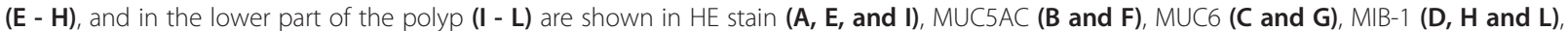
CDX2 (J), and MUC2 (K) immunostains. Surface epithelium in the upper part of the polyp is histologically similar to gastric foveolar epithelium and immunohistochemically positive for MUC5AC, suggesting gastric foveolar metaplasia (B), and the epithelium in the deeper portion and Brunner's glands are positive for MUC6 (C). This area shows high MIB-1 labeling (D). The similar staining pattern is observed in the mucosa with erosion (E - H). Surface epithelium in the lower part of the polyp shows a villous structure, containing CDX2-positive nuclei (J) and MUC2-positive goblet cells (K), suggesting preservation of the intestinal nature. MIB-1-positive Brunner's glands are inconspicuous in this area (L). All images are 40× magnification.
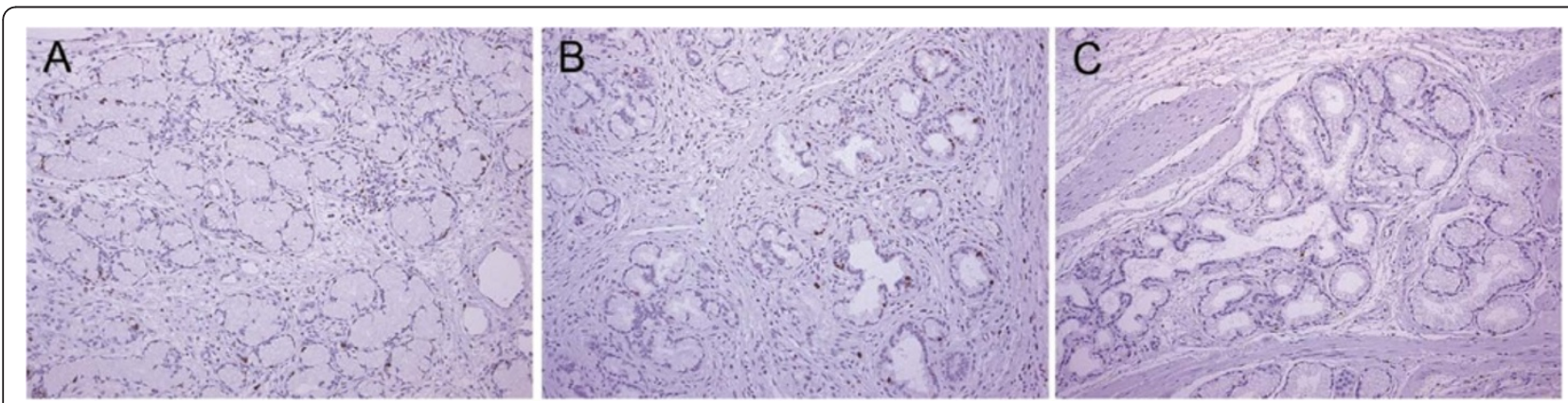

Figure 4 MIB-1 labeling in Brunner's glands. Representative immunostain images of Brunner's glands below surface epithelium associated with gastric foveolar metaplasia (A), below erosion (B), and below surface epithelium preserving intestinal nature (C) are shown. Brunner's glands in (C) are rarely positive for MIB-1, compared to those in (A) and (B). All images are 100x magnification. 
interchangeably called as Brunner's gland adenoma, however, some dispute using the latter because this lesion is usually an admixture of normal tissues without cytological atypia.

Brunner's gland hamartoma is predominantly found in those in their fifties to sixties. Several cases in their twenties have been reported with or without describing the lesion size, and to the best of our knowledge, only two cases (23- and 20-year-old women) have been reported mentioning their unusually large polyp size, $5.5 \mathrm{~cm}$ examined histologically and $7 \mathrm{~cm}$ observed by endoscopy $[5,6]$.

The major disadvantage having a large polyp is that a benign lesion may be misdiagnosed and over-treated as malignancy. In the past, a 60-year-old male case was reported that Brunner's gland hyperplasia forming a $5.5 \mathrm{~cm}$ polyp at duodenal bulb was treated by duodenocephalopancreatectomy [7]. When a large polyp is noted at duodenum, it is important for clinicians to have Brunner's gland hamartoma as a differential diagnosis, even with the rarity of the disease.

In our immunohistochemical study, proliferative activity of Brunner's glands was high beneath the epithelium showing gastric foveolar metaplasia. Gastric foveolar metaplasia seems to be a common finding in Brunner's gland hamartoma [2], and also associated with dysplastic changes in Brunner's gland hyperplasia [8,9]. One of the duodenal diseases frequently with gastric foveolar metaplasia is duodenal ulcer. In a duodenal ulcer lesion, mucosal repair mechanism initially generates epithelium with gastric foveolar metaplasia and then expands Brunner's glands [10]. Although how Brunner's gland hamartoma grows is unclear, it is reasonable to assume that repeated mucosal damages activate mucosal repair mechanism, facilitating a proliferation of Brunner's glands accompanied by surface gastric foveolar metaplasia.

Kim, et al., recently reported histology and mucin expression in Brunner's gland hamartoma and hyperplasia [2]. They showed that Brunner's gland hamartoma/hyperplasia lesions tended to be larger with surface epithelial change, such as erosion, ulcer, and gastric foveolar metaplasia, which is in accordance with our hypothesis that mucosal damage facilitates Brunner's gland proliferation. Although proliferative activity of Brunner's glands near surface epithelium was not described in their study, slightly increased proliferative activities in atypical glands and sclerotic glandular foci were reported with MIB-1 labeling index of $3 \%$ and $2 \%$, respectively. The sclerotic glandular foci are probably old lesions in the mucosal repair process, since the foci were found in $63.2 \%$ of the cases with surface epithelial changes but not in the cases without surface epithelial change [2].

Considering possible causes of mucosal damage, mechanical stimuli, Helicobacter pylori infection, and hyperacidic environment in duodenum have been suggested. The latter two have been postulated to be involved in the development of Brunner's gland hamartoma [11,12]. Helicobacter pylori infection was reported in some cases of Brunner's gland hamartoma [3], but not observed in the other cases [4]. The infection is unlikely to be essential for a hamartomatous proliferation of Brunner's glands. Hyperacidity can induce hyperplastic change of Brunner's glands, which secrete alkaline mucin to buffer gastric acid. Franzin, et al., demonstrated hyperacidic state in patients with Brunner's gland hamartoma [12]. Interestingly, gastric foveolar metaplasia has been regarded as a protective change of the duodenal mucosa against hyperacidity [13].

In summary, we report a case of large Brunner's gland hamartoma in a young (26-year-old) patient. Our immunohistochemical results suggest that mucosal damage underlies the hamartomatous proliferation of Brunner's glands.

\section{Consent}

Written informed consent was obtained from the patient for publication of this case report and any accompanying images. A copy of the written consent is available for review by the Series Editor of this journal.

\section{Abbreviations \\ MUC: Mucin; HE: Hematoxylin and eosin.}

\section{Competing interests}

The authors declare that they have no competing interests.

\section{Authors' contributions}

MA collected and analyzed the data, and was involved in drafting the manuscript. ST did clinical aspects of this study including surgical treatment. $\mathrm{KH}$ helped designing this study. RK guided us for pathological diagnosis and conception. HK revised the manuscript critically for important intellectual content and supported financially. All authors read and approved of the final manuscript.

\section{Acknowledgements}

We thank Ms. Keiko Hirano and Ms. Kana Sugita for technical assistance.

\section{Author details}

'Clinical Laboratory, University of Miyazaki Hospital, 5200 Kihara, Kiyotake, Miyazaki, Miyazaki 889-1692, Japan. ${ }^{2}$ Section of Oncopathology and Regenerative Biology, Department of Pathology, Faculty of Medicine, University of Miyazaki, 5200 Kihara, Kiyotake, Miyazaki, Miyazaki 889-1692, Japan. ${ }^{3}$ Section of Surgery, Koga General Hospital, 1749-1 Sudaki, Ikeuchi, Miyazaki, Miyazaki 880-0041, Japan. ${ }^{4}$ Section of Pathophysiology, Department of Pathology, Faculty of Medicine, University of Miyazaki, 5200 Kihara, Kiyotake, Miyazaki, Miyazaki 889-1692, Japan. ${ }^{5}$ Pathology Division, National Cancer Center Hospital, 5-1-1, Tsukiji, Chuo-ku, Tokyo 104-0045, Japan.

Received: 13 November 2012 Accepted: 10 January 2014

Published: 14 January 2014

\section{References}

1. Levine JA, Burgart LJ, Batts KP, Wang KK: Brunner's gland hamartomas: clinical presentation and pathological features of 27 cases. Am J Gastroenterol 1995, 90:290-294.

2. Kim K, Jang SJ, Song HJ, Yu E: Clinicopathologic characteristics and mucin expression in Brunner's gland proliferating lesions. Dig Dis Sci 2013, 58:194-201 
3. Kurella RR, Ancha HR, Hussain S, Lightfoot SA, Harty R: Evolution of Brunner gland hamartoma associated with Helicobacter pylori infection. South Med J 2008, 101:648-650.

4. Block KP, Frick TJ, Warner TF: Gastrointestinal bleeding from a Brunner's gland hamartoma: characterization by endoscopy, computed tomography, and endoscopic ultrasound. Am J Gastroenterol 2000, 95:1581-1583.

5. Vyas S, Skipworth JR, Lytras D, Smyth C, Mee A, Hatfield A, Imber C, Webster G: Rare presentation of Brunner's gland adenoma: another differentiation in patients with recurrent "idiopathic" pancreatitis. Hepatobiliary Pancreat Dis Int 2012, 11:107-110.

6. Stermer E, Elias N, Keren D, Rainis T, Goldstein O, Lavy A: Acute pancreatitis and upper gastrointestinal bleeding as presenting symptoms of duodenal Brunner's gland hamartoma. Can J Gastroenterol 2006, 20:541-542.

7. Iusco D, Roncoroni L, Violi V, Donadei E, Sarli L: Brunner's gland hamartoma: 'over-treatment' of a voluminous mass simulating a malignancy of the pancreatic-duodenal area. JOP 2005, 6:348-353.

8. Sakurai T, Sakashita H, Honjo G, Kasyu I, Manabe T: Gastric foveolar metaplasia with dysplastic changes in Brunner gland hyperplasia: possible precursor lesions for Brunner gland adenocarcinoma. Am J Surg Pathol 2005, 29:1442-1448.

9. Kushima R, Stolte M, Dirks K, Vieth M, Okabe H, Borchard F, Hattori T: Gastric-type adenocarcinoma of the duodenal second portion histogenetically associated with hyperplasia and gastric-foveolar metaplasia of Brunner's glands. Virchows Arch 2002, 440:655-659.

10. Kushima R, Manabe R, Hattori T, Borchard F: Histogenesis of gastric foveolar metaplasia following duodenal ulcer: a definite reparative lineage of Brunner's gland. Histopathology 1999, 35:38-43.

11. Kovacevic I, Ljubicic N, Cupic H, Doko M, Zovak M, Troskot B, Kujundzic M, Banic M: Helicobacter pylori infection in patients with Brunner's gland adenoma. Acta Med Croatica 2001, 55:157-160.

12. Franzin G, Musola R, Ghidini O, Manfrini C, Fratton A: Nodular hyperplasia of Brunner's glands. Gastrointest Endosc 1985, 31:374-378.

13. Ji R, Yu T, Gu XM, Zuo XL, An K, Zhou CJ, Li YQ: Gastric metaplasia of the duodenum: in vivo diagnosis by endomicroscopy and its relationship with functional dyspepsia. J Gastroenterol Hepatol 2011, 26:73-77.

doi:10.1186/1471-230X-14-14

Cite this article as: Akaki et al:: Duodenal mucosal damage is associated with proliferative activity of Brunner's gland hamartoma: a case report. BMC Gastroenterology 2014 14:14.

\section{Submit your next manuscript to BioMed Central and take full advantage of:}

- Convenient online submission

- Thorough peer review

- No space constraints or color figure charges

- Immediate publication on acceptance

- Inclusion in PubMed, CAS, Scopus and Google Scholar

- Research which is freely available for redistribution 